\title{
Plexin-D1/Semaphorin 3E pathway may contribute to dysregulation of vascular tone control and defective angiogenesis in systemic sclerosis
}

Celestina Mazzotta ${ }^{1 *}$, Eloisa Romano ${ }^{1}$, Cosimo Bruni ${ }^{1}$, Mirko Manetti ${ }^{2}$, Gemma Lepri ${ }^{1}$, Silvia Bellando-Randone ${ }^{1}$, Jelena Blagojevic ${ }^{1}$, Lidia Ibba-Manneschi ${ }^{2}$, Marco Matucci-Cerinic ${ }^{1}$ and Serena Guiducci ${ }^{1}$

\begin{abstract}
Introduction: The vascular and nervous systems have several anatomic and molecular mechanism similarities. Emerging evidence suggests that proteins involved in transmitting axonal guidance cues, including members of class III semaphorin (Sema3) family, play a critical role in blood vessel guidance during physiological and pathological vascular development. Sema3E is a natural antiangiogenic molecule that causes filopodial retraction in endothelial cells, inhibiting cell adhesion by disrupting integrin-mediated adhesive structures. The aim of the present study was to investigate whether in systemic sclerosis (SSc) Plexin-D1/Sema3E axis could be involved in the dysregulation of vascular tone control and angiogenesis.

Methods: Sema3E levels were measured by quantitative colorimetric sandwich ELISA in serum samples from 48 SSC patients, 45 subjects with primary Raynaud's phenomenon (pRP) and 48 age-matched and sex-matched healthy controls. Immunofluorescence staining on skin sections from 14 SSc patients and 12 healthy subjects was performed to evaluate Sema3E and Plexin-D1 expression. Western blotting was used to assess Plexin-D1/Sema3E axis in human SSc and healthy dermal microvascular endothelial cells (SSC-MVECs and H-MVECs, respectively) at basal condition and after stimulation with recombinant human vascular endothelial growth factor (VEGF), SSc and healthy sera. Capillary morphogenesis on Matrigel was performed on H-MVECs treated with healthy, pRP or SSc sera in the presence of Sema3E and Plexin-D1 soluble peptides.

Results: Serum Sema3E levels were significantly higher both in pRP subjects and SSc patients than in controls. In SSc, Sema3E levels were significantly increased in patients with early nailfold videocapillaroscopy (NVC) pattern compared to active/late patterns and pRP, and in patients without digital ulcers versus those with ulcers. In SSC skin, Sema3E expression was strongly increased in the microvascular endothelium. Cultured SSc-MVECs showed higher levels of phosphorylated Plexin-D1 and Sema3E expression than H-MVECS, and stimulation with SSc sera increased phosphorylated Plexin-D1 and Sema3E in H-MVECs. The addition of Sema3E-binding Plexin-D1 soluble peptide significantly attenuated the antiangiogenic effect of SSC sera on H-MVECS.
\end{abstract}

Conclusions: Our findings suggest that Plexin-D1/Sema3E axis is triggered in SSc endothelium and may have a role in the dysregulation of angiogenesis and vascular tone control by inducing neuro-vascular mechanism alterations clinically evident in particular in the early disease phases.

\footnotetext{
* Correspondence: celestina.mazzotta@unifi.it

'Department of Experimental and Clinical Medicine, Division of

Rheumatology, Azienda Ospedaliero-Universitaria Careggi (AOUC), University

of Florence, Viale Pieraccini 18, I-50139 Florence, Italy

Full list of author information is available at the end of the article
}

\section{Ciomed Central}

(c) 2015 Mazzotta et al. Open Access This article is distributed under the terms of the Creative Commons Attribution 4.0 International License (http://creativecommons.org/licenses/by/4.0/), which permits unrestricted use, distribution, and reproduction in any medium, provided you give appropriate credit to the original author(s) and the source, provide a link to the Creative Commons license, and indicate if changes were made. The Creative Commons Public Domain Dedication waiver (http://creativecommons.org/publicdomain/zero/1.0/) applies to the data made available in this article, unless otherwise stated. 


\section{Introduction}

Systemic sclerosis (SSc) is a multisystem autoimmune disease of unknown etiology characterized by vascular damage, activation of the immune system, and excessive deposition of collagen in the skin and internal organs including lungs, heart, gastrointestinal tract, and kidneys [1]. SSc is classified into limited cutaneous (lcSSc) and diffuse cutaneous (dcSSc) subsets and, in both, is associated with Raynaud's phenomenon (RP), nailfold capillaroscopic changes and antinuclear antibody positivity [2]. RP is characterized by recurrent, reversible episodes of vasospasm involving peripheral small vessels [3-5]. Early in the pathogenesis of RP, endothelial dysfunction increases platelet adhesion and favours dysfunctional control of vascular tone [6]. Endothelial cells are intimately involved in the regulation of vascular tone by synthesis and release of neurotransmitters, cytokines, growth factors, and prostaglandins, which mediate vasodilation and vasoconstriction [7]. Moreover, the vascular wall consists of endothelial, smooth muscle and fibroblast cells working in an integrated system of cellular paracrine/autocrine regulation interacting with the peripheral nervous system. The pathophysiology of RP is still not completely understood, but it has been suggested that dysregulation in neuro-endothelial control mechanisms plays a key role in RP pathogenesis [8].

Indeed, the vascular and nervous systems have several anatomical similarities that extend to molecular level, and the molecular mechanisms of nerve regulation are shared by the vascular system [9-12]. In some cases, vessels produce signals that attract axons to track alongside the pioneer vessels, conversely, nerves may also produce signals such as vascular endothelial growth factor (VEGF) to guide blood vessel growth. During embryogenesis, the developing nervous and vascular systems follow parallel routes and share several molecular pathways, including netrins, ephrins, and semaphorins, which act as both axon guidance molecules and regulators of developmental and postnatal angiogenesis $[9,13]$. Among them, secreted class III semaphorins (Sema3s) control endothelial cell migration in vitro and in vivo $[14,15]$. Semaphorins are grouped into eight classes based on their structural domains, and they are characterized by an amino-terminal Sema domain that is essential for signaling and can play a repulsive or attractive role depending on the cell types and biological context [16-18]. All Sema3 proteins (except Sema3E) signal through two major receptor families, namely Plexins and Neuropilins (NRPs), by forming a holoreceptor complex consisting of NRPs as ligand binding, and Plexins as signal transducing subunit. Conversely, Sema3E is unique in that it binds and signals directly through Plexin-D1 (PlxnD1), independently of NRPs. Plexins are single-pass transmembrane receptors subdivided into four groups, namely type A, B, C and D Plexins, with an extracellular Sema domain. Semaphorins target the actin cytoskeleton and focal adhesions, inducing cytoskeletal remodeling, cell motility, and cell migration $[19,20]$. Furthermore, it has been shown that Sema3E acts on PlxnD1 in endothelial cells to start an antiangiogenic signaling pathway [21], through the disassembly of integrin-mediated focal adhesions and consequent inhibition of endothelial cell adhesion to the extracellular matrix, ultimately leading to filopodia retraction in endothelial cells of growing blood vessels (Fig. 1) [22]. Moreover, PlxnD1 was shown to be highly expressed in angiogenic endothelial cells, and it was found to regulate the morphogenic patterning of developing vascular networks [13].

On these premises, the aims of this study were to investigate 1) the possible contribution of the antiangiogenic PlxnD1/Sema3E pathway in angiogenesis disturbances and development of capillary abnormalities and digital ulcers (DUs) during SSc [23-26], and 2) if this axis might participate in the dysregulation of vascular tone control in both SSc and primary RP (pRP).

\section{Methods}

\section{Study participants}

Serum samples were obtained from 48 patients with SSc (43 female and 5 male patients; median age 64 years, range 37 to 78 years), defined as limited cutaneous SSc $(\mathrm{lcSS} ; \mathrm{n}=36)$ or diffuse cutaneous SSc $(\mathrm{dcSSc} ; \mathrm{n}=12)$ [27], 45 subjects with pRP and 48 age-matched and sexmatched healthy controls. All SSc patients reported the occurrence of RP. Nailfold videocapillaroscopy (NVC) was performed on 10 fingers and all SSc patients were classified as having early, active and late NVC patterns [28]. At the time of blood collection, the presence of DUs was recorded. DUs related to trauma or calcinosis were not included in the analyses. The patients enrolled were not on corticosteroids, immunosuppressants or other disease-modifying drugs or calcium channel blockers. Peripheral blood samples were collected without any additive, left to clot for 30 minutes before centrifugation at $1,500 \mathrm{~g}$ for 15 minutes, and serum was collected and stored in aliquots at $-80{ }^{\circ} \mathrm{C}$ until used. Paraffin-embedded sections of lesional forearm skin biopsies were obtained from 14 patients with SSc (12 women and 2 men, $\mathrm{n}=9$ with lcSSc and $\mathrm{n}=5$ with dcSSc; median age 46.8 years, range 27 to 69 years, and median disease duration 6 years, range 1 to 16 years) and 12 age-matched and sex-matched healthy donors. The study was approved by the Ethical Committee of the Azienda Ospedaliero-Universitaria Careggi (AOUC), Florence, Italy, and all subjects provided written informed consent.

\section{Isolation of dermal microvascular endothelial cells (MVECs) and cell culture}

Human dermal MVECs were isolated from skin biopsies of five SSc patients (SSc-MVECs) and five healthy subjects 


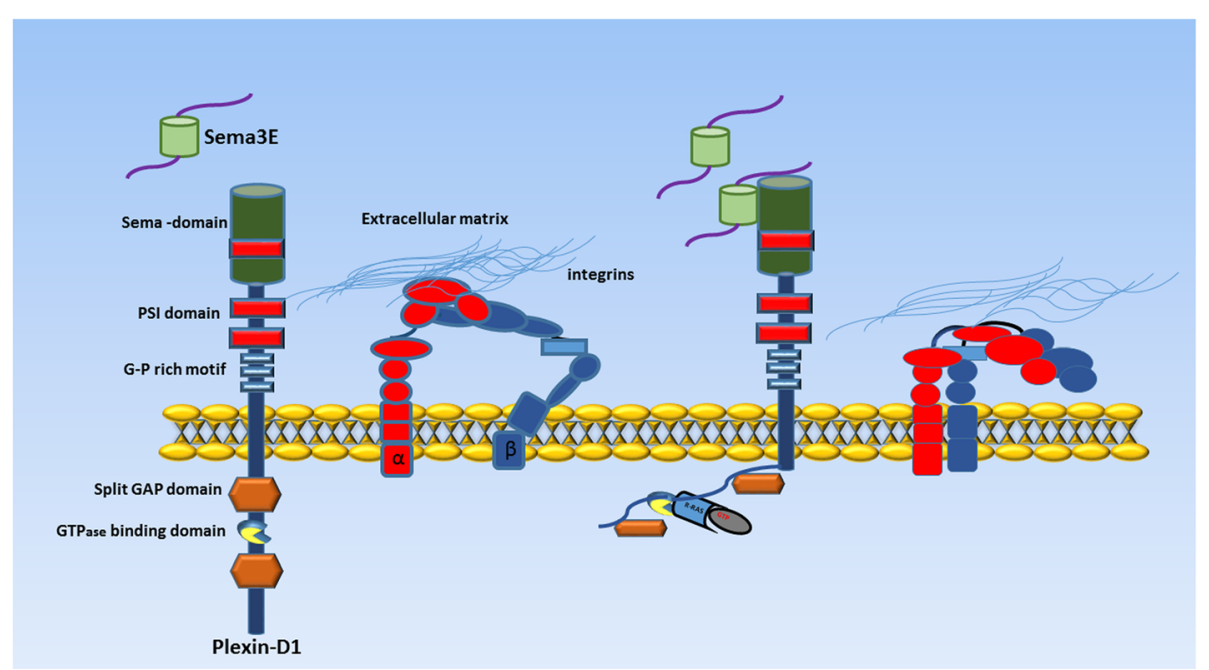

Fig. 1 Schematic representation of Plexin-D1/Semaphorin 3E (Sema3E) axis in endothelial cells. Binding of Sema3E to the cell surface receptor Plexin-D1 activates an antiangiogenic signaling pathway leading to the disassembly of integrin-mediated focal adhesions, inhibition of endothelial cell adhesion to the extracellular matrix, and filopodia retraction in endothelial cells of growing blood vessels

(H-MVECs). Briefly, the samples were mechanically cleaned to remove the adipose and epidermis layers, in order to obtain a pure specimen of vascularized dermis, and were treated as previously described [29]. The samples were placed at $37^{\circ} \mathrm{C}$ in a humidified atmosphere with $5 \% \mathrm{CO}_{2}$. After one day of culture in endothelial cell basal medium (EBM-2, catalog number LOCC3156; Euroclone, Milan, Italy) supplemented with $20 \%$ fetal bovine serum (FBS), $5 \mathrm{ng} / \mathrm{ml} \mathrm{H}$-epidermal growth factor (hEGF; Clonetics Corporation, San Diego, California, USA), $1 \mu \mathrm{g} / \mathrm{ml}$ hydrocortisone acetate, $100 \mathrm{U} / \mathrm{ml}$ penicillin, $100 \mu \mathrm{g} / \mathrm{ml}$ streptomycin, and $25 \mu \mathrm{g} / \mathrm{ml}$ amphotericin B without addition of further angiogenic growth factors, small colonies of polygonal elements were detected. Non-adherent cells were removed and fresh endothelial cell complete medium was added. To maintain optimal culture conditions, media were changed every third day, and after 2 weeks of primary culture a monolayer of cells was obtained. MVECs from primary cultures were further identified using immunomagnetic beads recognizing CD31. Isolated cells were purified as MVECs by labeling with anti-factor VIII-related antigen and antiCD105, followed by reprobing with anti-CD31 antibodies. Dermal MVECs were maintained in endothelial cell complete medium and were used between the third and seventh passages in culture.

\section{ELISA on serum samples}

Serum levels of Sema3E protein were measured by a colorimetric sandwich enzyme-linked immunosorbent assay (ELISA kit, catalogue number ABIN1117868; Antibodies Online, Aachen, Germany), according to the manufacturer's instructions. Briefly, standards and samples (100 $\mu \mathrm{l} /$ well) were added to the appropriate 96 -well microtiter plate pre-coated with a biotin-conjugated polyclonal antibody specific for the short basic Sema3E domain, and were incubated for 2 hours at $37{ }^{\circ} \mathrm{C}$. Subsequently the samples and standard were removed and $100 \mu \mathrm{l}$ of Detection Reagent A working solution were added to each well for 1 hour at $37{ }^{\circ} \mathrm{C}$. The microplates were washed three times with wash solution, followed by the addition of $100 \mu \mathrm{l}$ of Detection Reagent B working solution (Avidin-conjugated Horseradish Peroxidase (HRP)) to each well, and incubation for 30 minutes at $37{ }^{\circ} \mathrm{C}$. The microplates were washed five times and the reaction was developed in the dark with $90 \mu \mathrm{l}$ of substrate solution (tetramethylbenzidine) and then stopped by applying $50 \mu \mathrm{l}$ of sulfuric acid $\left(1 \mathrm{M} \mathrm{H}_{2} \mathrm{SO}_{4}\right)$. The absorbance of each well was read using a microplate reader at 450 $\mathrm{nm}$. Serum levels of Sema3E were read from a standard curve prepared using a lyophilized protein standard reconstituted with standard diluent included in the kit. The detection range of the assay was $0.156-20 \mathrm{ng} / \mathrm{ml}$. Each sample was measured in duplicate. Serum Sema3E concentration was determined by comparing the optical density (OD) of each sample to the standard curve.

\section{Immunohistochemistry and fluorescence microscopy}

Paraffin-embedded skin sections ( $5 \mu \mathrm{m}$ thick) were deparaffinized and boiled for 10 minutes in sodium citrate buffer (10 mM, pH 6.0) in order to expose antigens. After three washes in phosphate-buffered saline (PBS), the sections were incubated in $2 \mathrm{mg} / \mathrm{ml}$ glycine for 10 minutes to quench autofluorescence due to free aldehydes, and then blocked for 1 hour at room temperature with $1 \%$ bovine serum albumin (BSA) in PBS. The samples were then incubated overnight at $4{ }^{\circ} \mathrm{C}$ with goat anti human Sema3E 
antibody (catalog number ab112886; Abcam, Cambridge, UK) diluted 1:100 in PBS with $1 \%$ BSA. After extensive washing in PBS, the sections were incubated with Alexa Fluor-488-conjugated donkey anti-goat IgG for $45 \mathrm{mi}-$ nutes at room temperature in the dark (1:200 dilution; Invitrogen, San Diego, CA, USA). For double immunofluorescence staining, we used a rabbit polyclonal antibody against CD31/platelet-endothelial cell adhesion molecule1 (PECAM-1) diluted 1:50 (catalog number ab28364; Abcam), followed by Alexa Fluor-568-conjugated donkey anti-rabbit IgG (1:200 dilution; Invitrogen). To evaluate PlxnD1 expression, skin sections were incubated overnight at $4{ }^{\circ} \mathrm{C}$ with rabbit polyclonal antibody against human PlxnD1 (catalog number ab28762; Abcam) diluted 1:50 in PBS with $1 \%$ BSA. After extensive washing in PBS, the section were processed as described above, and subsequently incubated with Alexa Fluor-488-conjugated goat anti-rabbit IgG (1:200 dilution; Invitrogen) for $45 \mathrm{mi}-$ nutes at room temperature in the dark. For double immunofluorescence staining with anti-PlxnD1 antibody, we used a mouse monoclonal anti-CD31 antibody (1:20 dilution; catalog number M0823; Dako, Glostrup, Denmark) followed by Rhodamine Red-X-conjugated goat antimouse IgG (1:200 dilution; Invitrogen). Irrelevant isotypematched and concentration-matched goat, mouse, and rabbit IgG (Sigma-Aldrich, St Louis, MO, USA) were used as negative controls. Nuclei were counterstained with 4',6-diamidino-2-phenylindole (DAPI) (Chemicon International, Temecula, CA, USA). The immunolabeled sections were then observed under a Leica DM4000 B microscope equipped with fully automated fluorescence axes (Leica Microsystems, Mannheim, Germany). Fluorescence images were captured using a Leica DFC310 FX 1.4-megapixel digital colour camera equipped with the Leica software application suite LAS V3.8 (Leica Microsystems). Densitometric analysis of the intensity of immunofluorescent staining was performed on digitized images using the free-share ImageJ software $(\mathrm{NIH}, \mathrm{Be}-$ thesda, MD, USA; online at [30]).

\section{Western blotting and cell signaling}

Total proteins were extracted from dermal H-MVECs and SSc-MVECs according to standard protocols. In some experimental conditions, before protein extraction H-MVECs were cultured for 24 hours in EBM-2 medium containing $10 \%$ of serum from healthy subjects $(\mathrm{n}=5)$ and SSc patients $(\mathrm{n}=5)$ or recombinant human VEGF-A165 (10 ng/ml; R\&D Systems, Minneapolis, MN, USA). As determined by ELISA, healthy and SSc serum samples containing Sema3E levels similar to the respective median group value were used in these experiments. Twenty-five micrograms of total proteins were electrophoresed on NuPAGE 4 to $12 \%$ Bis-Tris Gel (Invitrogen) and were blotted on polyvinylidene difluoride membranes (Invitrogen).
The membranes were blocked for 40 minutes at room temperature with the blocking solution included in the Western Breeze Chromogenic Western Blot Immunodetection Kit (Invitrogen) on a rotary shaker and incubated for 1 hour at room temperature with rabbit polyclonal antihuman PlxnD1 (Sema domain $250 \mathrm{kDa}$, catalog number PP4401; 1:1000 dilution; ECM Biosciences, Versailles, Woodford County, KY, USA), rabbit polyclonal antihuman PlxnD1 (Cytoplasmic domain $250 \mathrm{kDa}$, catalog number PP4421; 1:500 dilution; ECM Biosciences), rabbit polyclonal antihuman PlxnD1 (a.a. 1635-1647 C-ter region $250 \mathrm{kDa}$, catalog number PP4441; 1:500 dilution; ECM Biosciences), rabbit polyclonal antihuman Sema3E (N-terminal region 87 $\mathrm{kDa}$, catalog number SP4461; 1:1000 dilution; ECM Biosciences) and rabbit polyclonal anti- $\alpha$-tubulin (catalog number ab18251; 1:1000 dilution; Abcam) antibodies, assuming $\alpha$ tubulin as control invariant protein. Immunodetection was performed as described in the Western Breeze Chromogenic Immunodetection protocol (Invitrogen). ImageJ software $(\mathrm{NIH})$ was used for densitometric analysis of the bands and all values were normalized to $\alpha$-tubulin.

\section{In vitro capillary morphogenesis assay}

In vitro capillary morphogenesis assay was performed in 96well plates covered with Matrigel (BD Biosciences, Milan, Italy). Matrigel $(50 \mu \mathrm{l} ; 10-12 \mathrm{mg} / \mathrm{ml})$ was pipetted into culture wells and polymerized for 30 minutes to 1 hour at $37{ }^{\circ} \mathrm{C}$, as described elsewhere [31]. H-MVECs and SScMVECs $\left(30 \times 10^{3}\right.$ cells/well $)$ were incubated in EBM-2 medium containing $10 \%$ FBS. In some experimental conditions, H-MVECs were incubated in EBM-2 medium with 10 $\%$ serum from healthy subjects $(n=5)$, SSc patients $(n=5)$ and pRP subjects $(\mathrm{n}=5)$, alone or in combination with human Sema3E peptide $(100 \mathrm{ng} / \mathrm{ml}$; catalog number ab39320; Abcam), human PlxnD1 peptide (a.a. 1683-1694) (100 ng/ml; catalog number ab45701; Abcam) or both. As determined by ELISA, healthy, pRP and SSc serum samples containing Sema3E levels similar to the respective median group value were used in these experiments. Stimulation with recombinant human VEGF-A165 (10 ng/ml; R\&D Systems) was used as positive control of angiogenesis. Plates were photographed at 6 and 24 hours. Results were quantified at 24 hours by measuring the percent field occupancy of capillary projections, as determined by image analysis. Six to nine photographic fields from three plates were scanned for each experimental point.

\section{Statistical analysis}

Data are expressed as the mean \pm standard deviation (SD) or median and range. The Student's $t$ test and nonparametric Mann-Whitney $U$ test were used where appropriate for statistical evaluation of the differences between two independent groups. A $p$ value less than 0.05 was considered statistically significant. 


\section{Results}

\section{Serum levels of Sema3E}

Sema3E serum levels were significantly increased both in pRP subjects (median $0.54 \mathrm{ng} / \mathrm{ml}$, range $0.00-1.96 \mathrm{ng} / \mathrm{ml}$ ) and SSc patients (median $0.67 \mathrm{ng} / \mathrm{ml}$, range $0.00-1.71 \mathrm{ng} /$ $\mathrm{ml}$ ) compared to controls (median $0.19 \mathrm{ng} / \mathrm{ml}$, range 0.00 $-0.94 \mathrm{ng} / \mathrm{ml}$ ) (both $p<0.005$ ) (Fig. 2a). No significant differences in circulating Sema3E levels were detected between pRP and SSc (Fig. 2a). Next, we correlated serum Sema3E levels with NVC patterns as measure of peripheral microvascular involvement. In SSc patients with early NVC pattern, Sema3E levels were significantly increased (median $1.17 \mathrm{ng} / \mathrm{ml}$, range $0.65-1.35 \mathrm{ng} / \mathrm{ml}$ ) compared with active (median $0.65 \mathrm{ng} / \mathrm{ml}$, range $0.00-1.71 \mathrm{ng} / \mathrm{ml}$ ) and late (median $0.55 \mathrm{ng} / \mathrm{ml}$, range $0.00-1.35 \mathrm{ng} / \mathrm{ml}$ ) NVC patterns (both $p<0.05$ ) (Fig. 2b). Moreover, serum Sema3E in SSc patients with early NVC pattern was significantly higher than in pRP subjects (median $0.54 \mathrm{ng} / \mathrm{ml}$, range $0.00-1.96 \mathrm{ng} / \mathrm{ml})(p<0.05)$. In addition, Sema3E levels were significantly increased in SSc patients without DUs (median $1.03 \mathrm{ng} / \mathrm{ml}$, range 0.00-1.71 ng/ml) compared with patients with DUs (median $0.57 \mathrm{ng} / \mathrm{ml}$, range $0.00-1.20 \mathrm{ng} / \mathrm{ml})(p=0.018)$ (Fig. 2c).

\section{Expression of Sema3E and PIxnD1 in skin biopsies}

The expression of Sema3E and its receptor PlxnD1 in skin biopsies from SSc patients and healthy controls was evaluated by immunofluorescence (Figs. 3 and 4). Sema3E expression was strongly increased in several cellular components of SSc dermis, such as fibroblasts and perivascular cells when compared with healthy controls (Fig. 3a, b). Moreover, double immunofluorescence staining for Sema $3 \mathrm{E}$ and the pan-endothelial cell marker CD31/PECAM-1 revealed that Sema3E expression was significantly upregulated in endothelial cells of SSc dermis compared with healthy control skin (Fig. 3c

-f). The densitometric analysis of the intensity of immunofluorescent staining demonstrated that Sema3E expression was significantly increased in the dermis of $\mathrm{SSc}$ patients compared with controls $(p<0.001)$ (Fig. 3g). Conversely, no significant difference in the expression of PlxnD1 was observed between healthy skin and skin from patients with SSc in different dermal cell types, including endothelial cells (Fig. 4a-g).

\section{PIxnD1/Sema3E cell signaling in dermal MVECs}

To assess the role of PlxnD1/Sema3E pathway in the endothelium, we evaluated cell signaling on H-MVECs cultured in standard condition and after challenge with recombinant human VEGF-A165 and sera from healthy subjects or patients with SSc, and in SSc-MVECs cultured in standard conditions. Western blotting analyses revealed that SSc-MVECs expressed significant higher levels of the activated form of PlxnD1 (phosphorylated in
C-ter domain, a.a. 1635-1647) than H-MVECs $(p<0.001)$ (Fig. 5a). In addition, the phosphorylated form of PlxnD1 significantly increased in H-MVECs challenged with sera from patients with SSc compared with both basal $\mathrm{H}$ MVECs and H-MVECs cultured with sera from healthy controls (both $p<0.05$ ) (Fig. 5a). To validate these results, we also specifically evaluated the expression levels of the $\mathrm{N}$-terminal Sema domain region of PlxnD1, which can be detected only when this domain is not bound by Sema3E. As shown in Fig. 5b, the detection levels of this region were significantly lower in SSc-MVECs than in $\mathrm{H}$ MVECs, and in H-MVECs challenged with sera from patients with SSc compared with H-MVECs treated with sera from healthy controls (both $p<0.05$ ). Moreover, consistent with the immunohistochemical results, Sema3E levels were significantly increased in SSc-MVECs in respect to H-MVECs, and in H-MVECs challenged with sera from patients with SSc compared with H-MVECs treated with sera from healthy controls (both $p<0.05$ ) (Fig. 5c). No significant differences in total PlxnD1 expression were found between the different experimental conditions (Fig. 5d). Finally, the stimulation with recombinant human VEGF-A165 did not significantly affect the PlxnD1/Sema3E pathway in H-MVECs (Fig. 5a-d).

\section{Capillary morphogenesis on Matrigel}

To investigate the functional influence of the PlxnD1/ Sema3E pathway on dermal MVEC in vitro angiogenesis, we performed capillary morphogenesis on Matrigel matrix. In this assay, MVECs usually produce elongated processes that eventually form anastomosing cords of cells mimicking a tubular capillary plexus. Consistent with previous findings [31], in standard conditions angiogenesis was strongly impaired in SSc-MVECs compared with H-MVECs (Fig. 6). H-MVECs stimulated with healthy sera produced an abundant network of branching cords, while the addition of Sema3E soluble peptide to the sera significantly inhibited angiogenesis ( $p<0.05$ vs healthy serum only) (Fig. 6). Furthermore, the angiogenic capacity of H-MVECs coincubated with healthy sera and Sema3E-binding PlxnD1 peptide or Sema3E/PlxnD1 combined peptides was not different compared to that of cells treated with healthy sera alone (Fig. 6). Similar results were obtained when H-MVECs were challenged with sera from patients with pRP, except that co-incubation with pRP serum and Sema3E-binding PlxnD1 peptide significantly increased angiogenesis compared to treatment with serum from pRP alone $(p<0.05)$ (Fig. 6). Moreover, capillary morphogenesis was reduced in $\mathrm{H}$ MVECs challenged with sera from patients with pRP compared with cells treated with healthy sera, but this difference was not statistically significant. Conversely, treatment with sera from patients with SSc strongly 


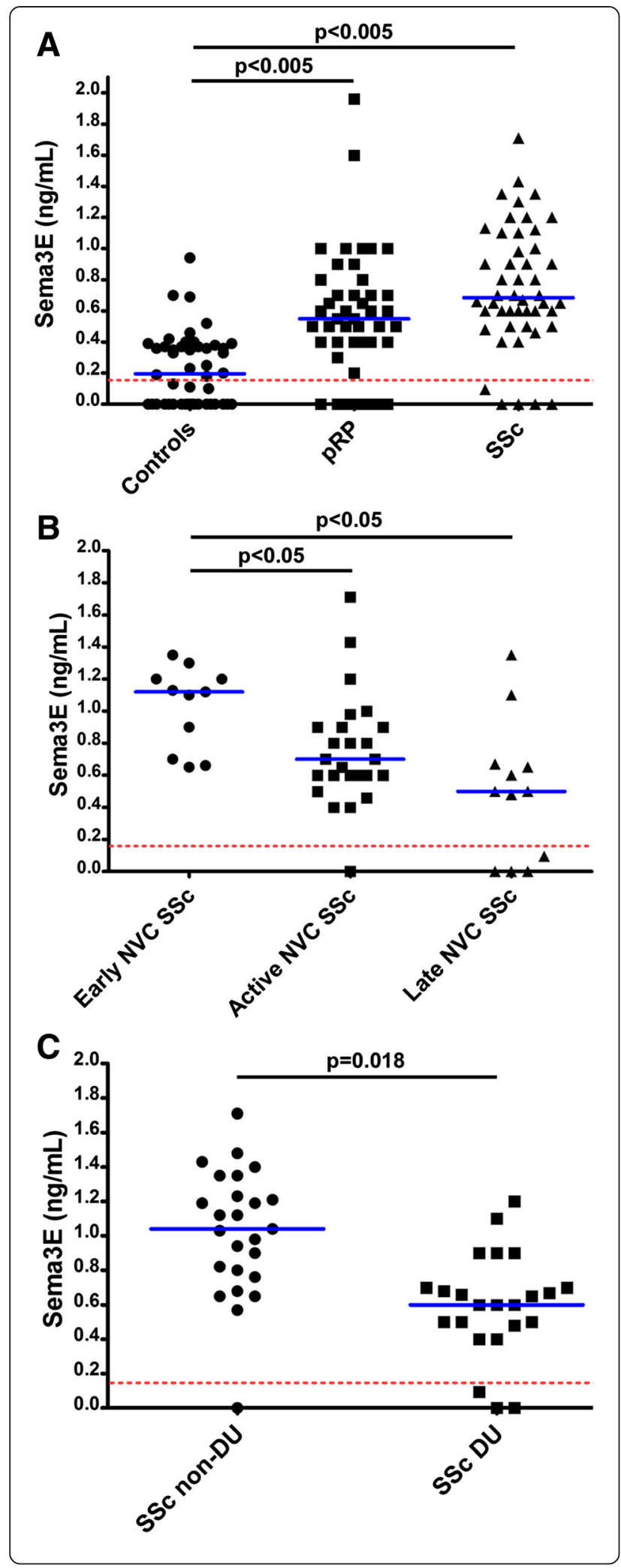

Fig. 2 Serum levels of semaphorin 3E (Sema3E) determined by colorimetric sandwich enzyme-linked immunosorbent assay (ELISA). a Serum Sema3E levels in healthy controls, subjects with primary Raynaud's phenomenon ( $p R P)$ and patients with systemic sclerosis (SSc). b Serum Sema3E levels in SSc patients according to nailfold videocapillaroscopy (NVC) pattern (early, active and late). c Serum Sema3E levels in SSc patients according to the absence/presence of digital ulcers (DUs). Data are shown as dot plots. Each dot represents a subject. Blue horizontal lines indicate the median value in each group. In each panel, the red dashed line indicates the detection threshold of the ELISA. The nonparametric Mann-Whitney $U$ test for independent samples was used to analyze the serum Sema3E differences between groups. Values of $p<0.05$ were considered significant; $p$ values are indicated in each panel

reduced angiogenesis compared with healthy sera (Fig. 6). The addition of Sema3E peptide further impaired the angiogenic capacity of H-MVECs challenged with sera from patients with SSc $(p<0.05$ vs serum from patients with SSc only) (Fig. 6). On the contrary, when H-MVECs were co-incubated with sera from patients with SSc and Sema3E-binding PlxnD1 soluble peptide, their angiogenic capacity was significantly increased compared with cells treated with sera from patients with SSc alone $(p<0.05)$ (Fig. 6).

\section{Discussion}

Our data show for the first time that 1) serum levels of Sema3E are significantly increased both in pRP subjects and SSc patients with secondary RP, and that 2) the PlxnD1/Sema3E signaling pathway is triggered in SScMVECs and determines an antiangiogenic response.

Blood vessels provide oxygen and nutrients to every part of the body, and they are essential for tissue homeostasis and repair. Angiogenesis is a physiological process where new vessels are formed by sprouting of endothelial cells from pre-existent vessels to create and increase a complex network [32-35]. Angiogenesis is controlled by a tight and complex balance between proangiogenic and antiangiogenic signals, and among them VEGF coordinates the angiogenesis process. Moreover, the endothelium produces a large number of vasodilating factors, including nitric oxide and prostacyclin [36], and vasoconstricting substances, such as endothelin-1, with effects on vascular remodeling [37]. In this context, increasing evidence indicates that both $\mathrm{pRP}$ and secondary RP in SSc are characterized by severe disturbances in multiple mechanisms that regulate vascular tone control $[2,24]$. Moreover, an impaired angiogenic response following tissue ischemia and hypoxia is considered a hallmark feature of SSc [24].

Of note, it is well-established that the autonomic nervous system contributes to the pathogenesis of both $\mathrm{pRP}$ and secondary RP in SSc, via central and peripheral 

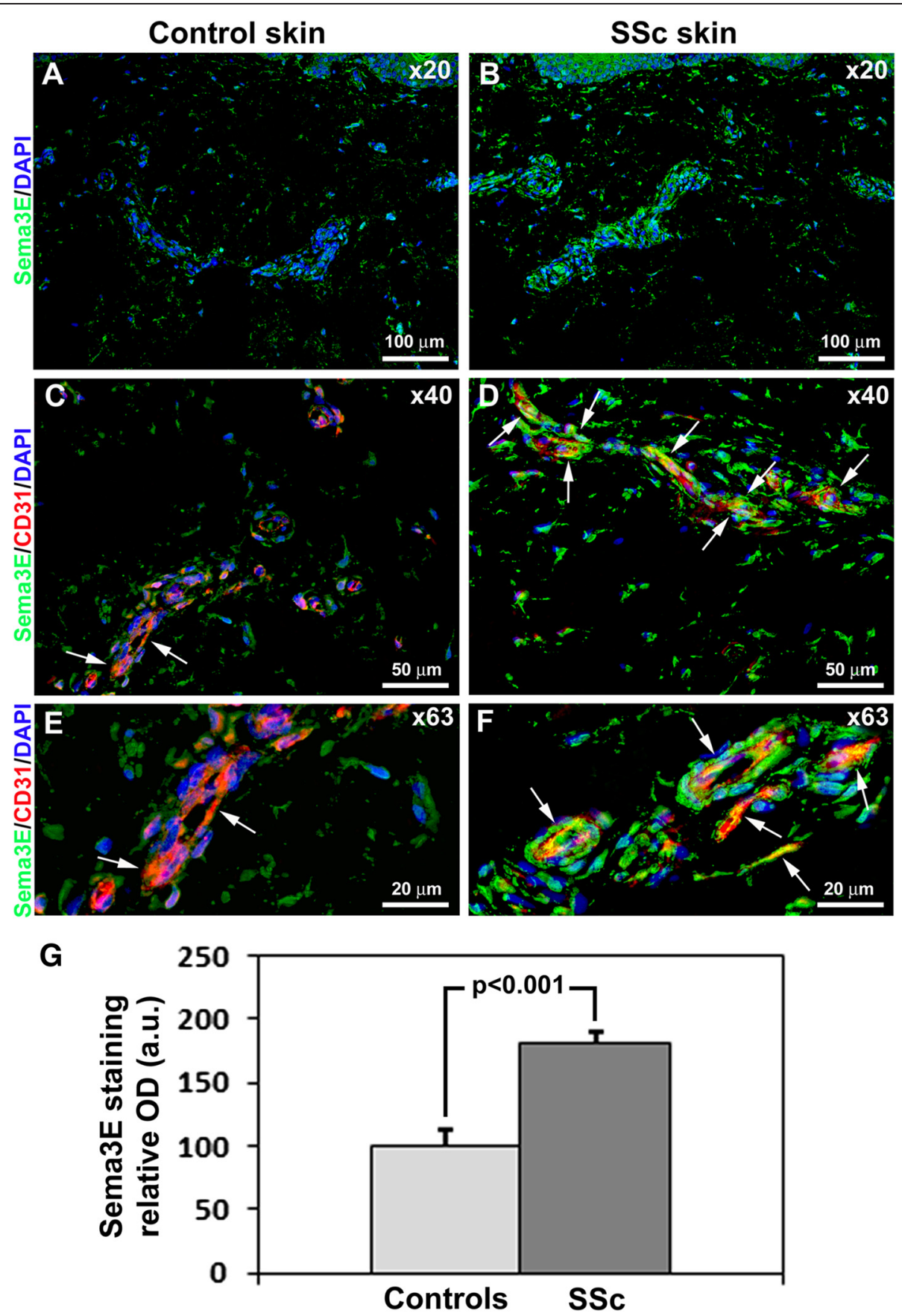

Fig. 3 Expression of semaphorin $3 E$ (Sema3E) in skin of healthy controls and patients with systemic sclerosis (SSC). a, b Representative microphotographs of skin sections from healthy controls $(\mathbf{a})(\mathrm{n}=12)$ and patients with SSc $(\mathbf{b})(\mathrm{n}=14)$ immunostained for Sema3E (green) and counterstained with 4',6-diamidino-2-phenylindole (DAPl; blue) for nuclei. c-f Representative microphotographs of skin sections from healthy controls $(\mathbf{c}, \mathbf{e})(n=12)$ and patients with SSC $(\mathbf{d}, \mathbf{f})(n=14)$ double immunostained for Sema3E (green) and CD31/platelet-endothelial cell adhesion molecule-1 (PECAM-1) (red) and counterstained with DAPI (blue). Arrows indicate Sema3E expression in dermal microvascular endothelial cells. Original magnification: $\times 20(\mathbf{a}, \mathbf{b}), \times 40(\mathbf{c}, \mathbf{d}), \times 63(\mathbf{e}, \mathbf{f})$. Scale bars are indicated in each panel. $\mathbf{g}$ Densitometric analysis of Sema3E immunofluorescent staining in skin biopsies expressed as optical density (OD) in arbitrary units (a.u.). Control skin OD value was set to $100 \%$; the other results are normalized to this value. Data are mean \pm SD. Student's $t$ test was used for statistical analysis

mechanisms [38]. Furthermore, the vascular and nervous systems have several morphological and molecular similarities $[9,19,35]$. In fact, emerging evidence suggests that proteins involved in transmitting axonal guidance cues, such as the multiple members of secreted class III semaphorin family that regulate developmental axonal 

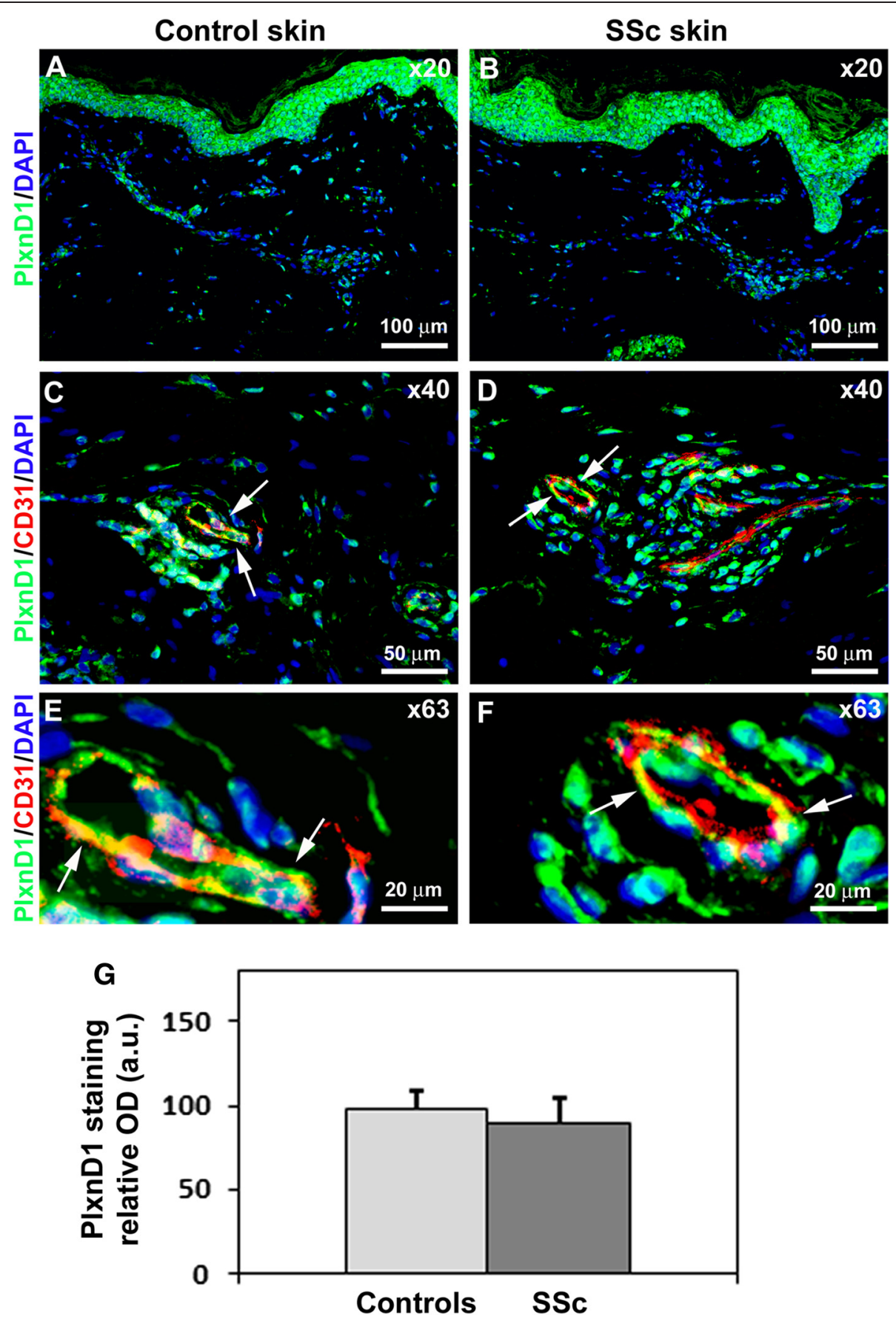

Fig. 4 Expression of Plexin-D1 (PIxnD1) in skin of healthy controls and patients with systemic sclerosis (SSC). a, b Representative microphotographs of skin sections from healthy controls $(\mathbf{a})(n=12)$ and SSc patients $(\mathbf{b})(n=14)$ immunostained for PlxnD1 (green) and counterstained with 4',6-diamidino-2-phenylindole (DAPl; blue) for nuclei. $\mathbf{c}$-f Representative microphotographs of skin sections from healthy controls $(\mathbf{c}, \mathbf{e})(\mathrm{n}=12)$ and SSc patients $(\mathbf{d}, \mathbf{f})(\mathrm{n}=14)$ double immunostained for PIxnD1 (green) and CD31/platelet-endothelial cell adhesion molecule-1 (PECAM-1) (red) and counterstained with DAPI (blue). Arrows indicate PIxnD1 expression in dermal microvascular endothelial cells. Original magnification: $\times 20(\mathbf{a}, \mathbf{b}), \times 40(\mathbf{c}, \mathbf{d}), \times 63(\mathbf{e}, \mathbf{f})$. Scale bars are indicated in each panel. $\mathbf{g}$ Densitometric analysis of PlxnD1 immunofluorescent staining in skin biopsies expressed as optical density (OD) in arbitrary units (a.u.). Control skin OD value was set to $100 \%$; the other results are normalized to this value. Data are mean \pm SD. Student's $t$ test was used for statistical analysis

growth, also play a critical role in blood vessel guidance during physiological and pathological vascular development $[9,39,40]$. In particular, soluble Sema3E is a potent inhibitor of angiogenesis that binds to its specific receptor PlxnD1 with consequent activation of a complex antiangiogenic intracellular signaling cascade [23]. 


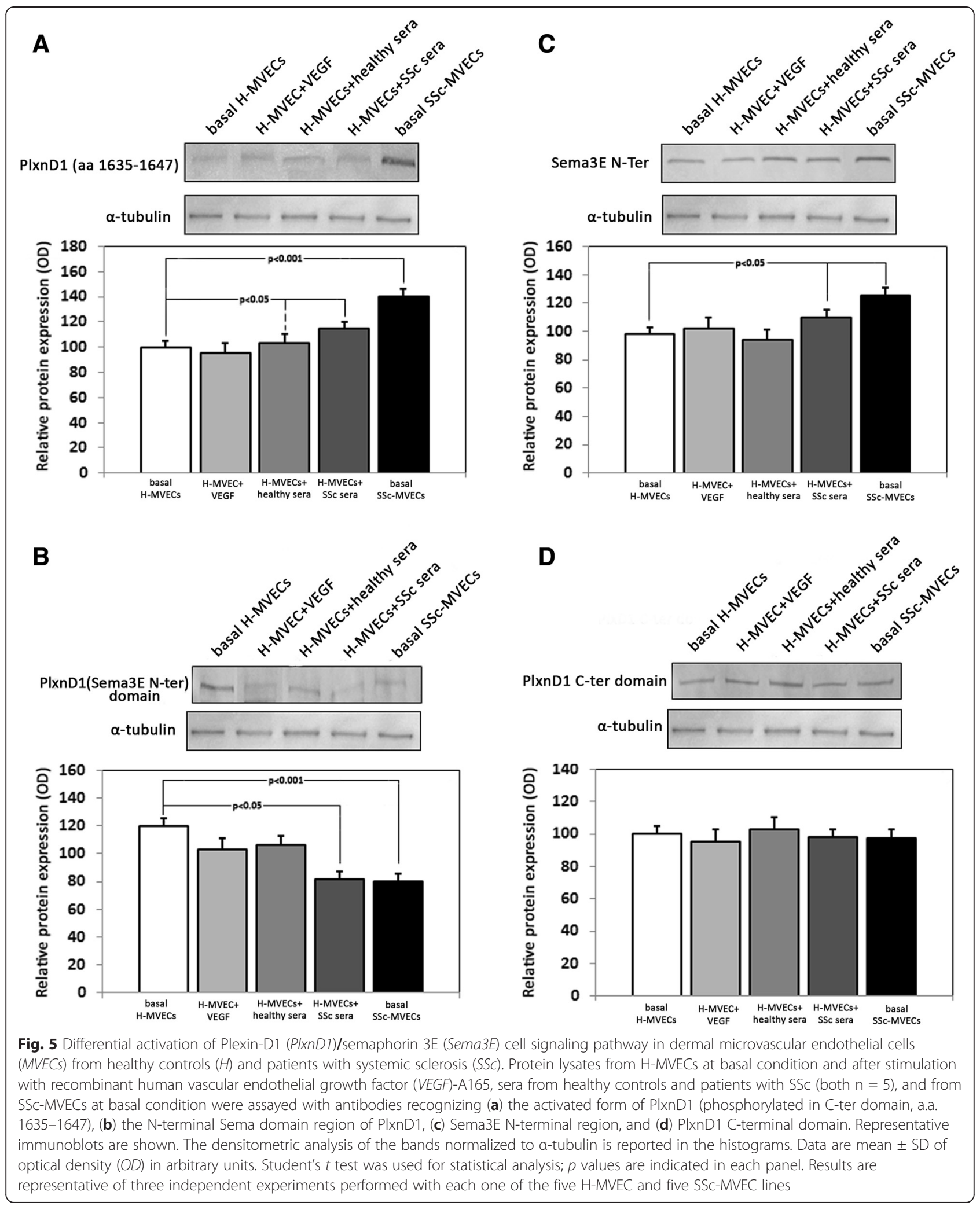

The present study was undertaken to investigate whether the PlxnD1/Sema3E axis could be involved in the neurovascular component of SSc pathophysiology.
For this purpose, we first analyzed circulating levels of Sema3E both in pRP subjects and SSc patients, as well as their possible correlation with measures of vascular 


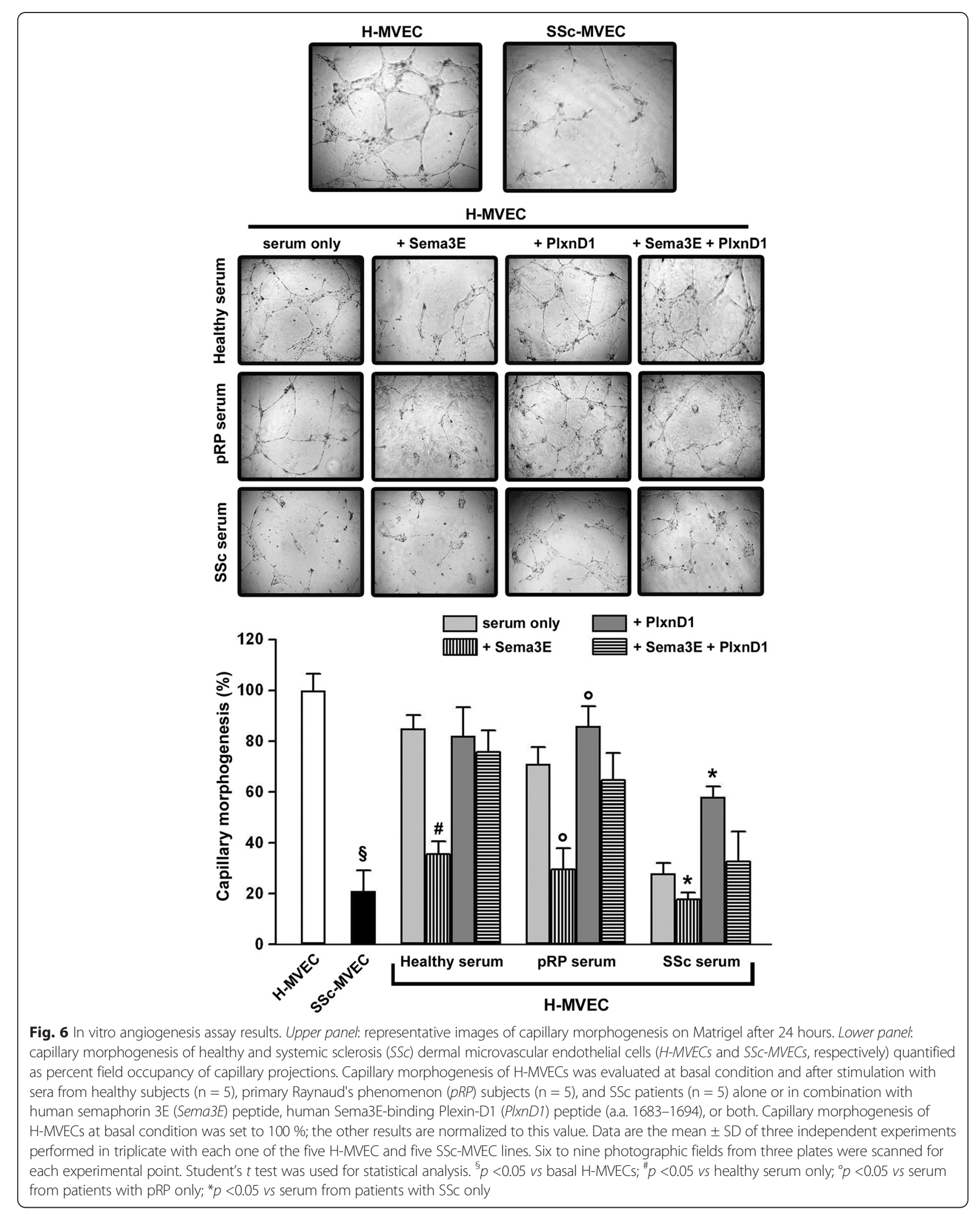


involvement in SSc. Interestingly, we found significantly increased serum Sema3E levels both in pRP and SSc compared with healthy individuals, suggesting that this molecule might participate in the vascular tone disturbances characteristic of both clinical entities. Moreover, it is interesting to note that in SSc increased circulating levels of Sema3E specifically correlated with the early NVC pattern and the absence of DUs, as patients with more severe capillary damage and DUs had Sema3E levels comparable to those of controls. Thus, it is tempting to speculate that Sema3E might even serve in the future as a biomarker of early vascular involvement during SSc. Indeed, currently there are no reliable serological biomarkers for the early diagnosis of RP and the neuro-vascular involvement in SSc patients. However, further follow-up studies will be necessary to ascertain whether circulating Sema3E could be a useful marker to monitor the evolution of SSc-related peripheral vascular disease. Furthermore, we cannot exclude the possibility that lower Sema3E levels detected in SSc with more advanced NVC patterns might be either a cause or a consequence of the disease, which is characterized by progressive loss of the peripheral microvessels and nervous fibers [41], which are the main source of Sema3E.

Consistent with serum findings, the expression of Sema3E in SSc-affected dermis was strongly increased, particularly in the microvascular endothelium. Conversely, no difference in the expression of Sema3E receptor PlxnD1 was found between skin from patients with SSc and healthy controls. To further address whether the PlxnD1/Sema3E pathway could be activated in the endothelium in SSc, we performed cell signaling studies on cultured dermal MVECs. Strikingly, our in vitro experiments demonstrated that although total PlxnD1 expression was not different in H-MVECs and SSc-MVECs, these latter showed a significantly increase in the expression of the activated form of PlxnD1 (phosphorylated in C-ter domain). Moreover, phosphorylated PlxnD1 significantly increased in H-MVECs after challenge with sera from patients with SSc. Thus, these data support the hypothesis that PlxnD1/ Sema3E pathway is triggered in the microvascular endothelium in SSc. Finally, functional capillary morphogenesis experiments revealed that such activation of the PlxnD1/ Sema3E pathway may contribute to defective angiogenesis in SSc. Indeed, we showed that Sema3E exerts antiangiogenic effects in vitro. Accordingly, serum from patients with SSc strongly inhibited angiogenesis of H-MVECs, while the addition of Sema3E-binding PlxnD1 soluble peptide to sequester elevated Sema3E present in sera from patients with SSc significantly improved H-MVEC angiogenesis.

\section{Conclusions}

In conclusion, our findings suggest that the PlxnD1/ Sema3E axis is triggered in the endothelium in SSc, and may have a role in the dysregulation of angiogenesis and vascular tone control by inducing neurovascular mechanism alterations, which are clinically evident in particular in the early disease phases.

\begin{abstract}
Abbreviations
a.u.: arbitrary units; BSA: bovine serum albumin; DAPI: 4',6-diamidino-2phenylindole; dcSSc: diffuse cutaneous systemic sclerosis; DU: digital ulcer; EBM: endothelial cell basal medium; ELISA: enzyme-linked immunosorbent assay; H-MVECs: human dermal microvascular endothelial cells from healthy subjects; IgG: immunoglobulin G; ICSSc: limited cutaneous systemic sclerosis; MVECs: microvascular endothelial cells; NRP: neuropilin; NVC: nailfold videocapillaroscopy; OD: optical density; PBS: phosphate-buffered saline; PECAM-1: platelet-endothelial cell adhesion molecule-1; PlxnD1: Plexin-D1; pRP: primary Raynaud's phenomenon; RP: Raynaud's phenomenon; SD: standard deviation; Sema3E: semaphorin 3E; SSc: systemic sclerosis; SScMVECs: human dermal microvascular endothelial cells from patients with systemic sclerosis; VEGF: vascular endothelial growth factor.
\end{abstract}

\section{Competing interests}

The authors declare that they have no competing interests.

\section{Authors' contributions}

All authors meet the criteria for authorship. CM conceived the study, participated in its design and coordination, contributed to most of the experiments, analysis and interpretation of data, drafted and edited the manuscript and revised it critically for important intellectual content. ER contributed to dermal MVEC isolation and culture, ELISA, Western blotting experiments, analysis and interpretation of data and helped to draft the manuscript. CB collected and supplied biological samples and clinical data and helped to draft the manuscript. MM contributed to dermal MVEC isolation, immunofluorescence experiments and analysis of data, drafted and edited the manuscript and revised it critically for important intellectual content. GL, SB-R and JB collected and supplied serum samples and clinical data and helped to revise the manuscript. LI-M contributed to the immunofluorescence experiments and analysis of data and helped to draft the manuscript. MM-C participated in study design and coordination and interpretation of data, drafted the article and revised it critically for important intellectual content. SG participated in study coordination, contributed to dermal MVEC isolation and helped to draft and revise the manuscript. All authors approved the final version of the manuscript to be published.

\section{Acknowledgements}

The authors gratefully acknowledge Irene Rosa (Department of Experimental and Clinical Medicine, University of Florence, Florence, Italy) for the excellent technical support in immunofluorescence studies.

\section{Author details}

${ }^{1}$ Department of Experimental and Clinical Medicine, Division of Rheumatology, Azienda Ospedaliero-Universitaria Careggi (AOUC), University of Florence, Viale Pieraccini 18, I-50139 Florence, Italy. ${ }^{2}$ Department of Experimental and Clinical Medicine, Section of Anatomy and Histology, University of Florence, Largo Brambilla 3, I-50134 Florence, Italy.

Received: 23 February 2015 Accepted: 10 August 2015

Published online: 21 August 2015

\section{References}

1. Abraham DJ, Varga J. Scleroderma: from cell and molecular mechanisms to disease models. Trends Immunol. 2005;26:587-95.

2. Wigley FM. Raynaud's Phenomenon. N Engl J Med. 2002;347:1001-8.

3. Noel B. Pathophysiology and classification of the vibration white finger. Int Arch Occup Environ Health. 2000;73:150-5.

4. Doll DC, Yarbro JW. Vascular toxicity associated with antineoplastic agents. Semin Oncol. 1992;19:580-96.

5. Boin F, Wigley FM. Understanding, assessing and treating Raynaud's phenomenon. Curr Opin Rheumatol. 2005;17:752-60.

6. Herrick AL. Diagnosis and management of scleroderma peripheral vascular disease. Rheum Dis Clin North Am. 2008;34:89-114. 
7. Wigley FM, Flavahan NA. Raynaud's phenomenon. Rheum Dis Clin N Am. 1996;22:765-81.

8. Kaheleh B, Matucci-Cerinic M. Raynaud's phenomenon and scleroderma. Dysregulated neuroendothelial control of vascular tone. Arthritis Rheum. 1995;38:1-4.

9. Carmeliet P, Tessier-Lavigne M. Common mechanisms of nerve and blood vessel wiring. Nature. 2005;436:193-200.

10. Dickson BJ. Molecular mechanisms of axon guidance. Science. 2002;298:1959-64.

11. Weinstein BM. Vessels and nerves: marching to the same tune. Cell. 2005;120:299-302.

12. Eichmann A, Le Noble F, Autiero M, Carmeliet P. Guidance of vascular and neural network formation. Curr Opin Neurobiol. 2005;15:108-15.

13. Suda K, Guo C, Oishi A, Ikeda S, Uemura A, Yoshimura N. Therapeutic potential of semaphorin $3 \mathrm{E}$ for the treatment of choroidal neovascularization. Invest Ophthalmol Vis Sci. 2014;55:4700-6.

14. Serini G, Valdembri D, Zanivan S, Morterra G, Burkhardt C, Caccavari F, et al. Class 3 semaphorins control vascular morphogenesis by inhibiting integrin function. Nature. 2003;424:391-7.

15. Kessler O, Shraga-Heled N, Lange T, Gutmann-Raviv N, Sabo E, Baruch L, et al. Semaphorin-3F is an inhibitor of tumor angiogenesis. Cancer Res. 2004;64:1008-115.

16. Eichmann A, Makinen T, Alitalo K. Neural guidance molecules regulate vascular remodeling and vessel navigation. Genes Dev. 2005;19:1013-21.

17. Derijck AA, Van Erp S, Pasterkamp RJ. Semaphorin signaling: molecular switches at the midline. Trends Cell Biol. 2010;20:568-76.

18. Gu C, Yoshida Y, Livet J, Reimert DV, Mann F, Merte J, et al. Semaphorin 3E and plexin-D1 control vascular pattern independently of neuropilins. Science. 2005;307:265-8.

19. Gelfand MV, Hong S, Gu C. Guidance from above: common cues direct distinct signaling outcomes in vascular and neural patterning. Trends Cell Biol. 2009;19:99-110

20. Serini G, Napione L, Arese M, Bussolino F. Besides adhesion: new perspectives of integrin functions in angiogenesis. Cardiovasc Res. 2008;78:213-22.

21. Sakurai A, Gavard J, Annas-Linhares Y, Basile JR, Amornphimoltham P, Palmby $T R$, et al. Semaphorin 3E initiates antiangiogenic signaling through plexin D1 by regulating Arf6 and R-Ras. Mol Cell Biol. 2010;30:3086-98.

22. Brown FD, Rozelle AL, Yin HL, Balla T, Donaldson JG. Phosphatidylinositol 4,5-bisphosphate and Arf6-regulated membrane traffic. J Cell Biol. 2001;154:1007-17.

23. Varga J, Abraham D. Systemic sclerosis: a prototypic multisystem fibrotic disorder. J Clin Invest. 2007;15:557-67.

24. Matucci-Cerinic M, Kahaleh B, Wigley FM. Review: evidence that systemic sclerosis is a vascular disease. Arthritis Rheum. 2013;15:1953-62.

25. Khimdas S, Harding S, Bonner A, Zummer B, Baron M, Pope J. Associations with digital ulcers in a large cohort of systemic sclerosis: results from the Canadian Scleroderma Research Group registry. Arthritis Care Res (Hoboken). 2011;63:142-9.

26. Kuwana M, Okazaki Y, Yasuoka H, Kawakami Y, Ikeda Y. Defective vasculogenesis in systemic sclerosis. Lancet. 2004;364:603-10.

27. LeRoy EC, Black C, Fleischmajer R, Jablonska S, Krieg T, Medsger Jr TA, et al Scleroderma (systemic sclerosis): classification, subsets, and pathogenesis. J Rheumatol. 1988;15:202-5.

28. van den Hoogen F, Khanna D, Fransen J, Johnson SR, Baron M, Tyndall A, et al. Classification Criteria for Systemic Sclerosis: An American College of Rheumatology/European League Against Rheumatism Collaborative Initiative. Arthritis Rheum. 2013;2013:2737-47.

29. Scott PA, Bicknell R. The isolation and culture of microvascular endothelium. J Cell Sci. 1993:105:269-73.

30. ImageJ. http://rsbweb.nih.gov/ij.

31. Manetti M, Guiducci S, Romano E, Ceccarelli C, Bellando Randone S, Conforti ML, et al. Overexpression of VEGF165b, an inhibitory splice variant of vascular endothelial growth factor, leads to insufficient angiogenesis in patients with systemic sclerosis. Circ Res. 2011;109:e14-26.

32. Risau W. Mechanisms of angiogenesis. Nature. 1997;386:671-4.

33. Gerhardt H. VEGF and endothelial guidance in angiogenic sprouting Organogenesis. 2008;4:241-6.

34. De Smet F, Segura I, De Bock K, Hohensinner PJ, Carmeliet P. Mechanisms of vessel branching: filopodia on endothelial tip cells lead the way. Arterioscler Thromb Vasc Biol. 2009:29:639-49.
35. Adams RH, Eichmann A. Axon guidance molecules in vascular patterning. Cold Spring Harb Perspect Biol. 2010;2:a001875.

36. Tucker AT, Pearson RM, Cooke ED, Benjamin N. Effect of nitric-oxidegenerating system on microcirculatory blood flow in skin in patients with severe Raynaud's syndrome: a randomized trial. Lancet. 1999;354:1670-5.

37. Kirchengast M, Munter K. Endothelin-1 and endothelin receptor antagonists in cardiovascular remodeling. Proc Soc Exp Biol Med. 1999;221:312-5.

38. Herrick AL, Clements PJ, Furst DE. Nervous system In Systemic sclerosis. 2nd ed. Philadelphia: Williams \& Wilkins; 2004. p. 309-17.

39. Raper JA. Semaphorins and their receptors in vertebrates and invertebrates. Curr Opin Neurobiol. 2000;10:88-94.

40. Tessier-Lavigne M, Goodman CS. The molecular biology of axon guidance. Science. 1996:274:1123-33.

41. Ibba-Manneschi L, Niissalo S, Milia AF, Allanore Y, Del Rosso A, Pacini A, et al. Variations of neuronal nitric oxide synthase in systemic sclerosis skin. Arthritis Rheum. 2006;54:202-13.

\section{Submit your next manuscript to BioMed Central and take full advantage of:}

- Convenient online submission

- Thorough peer review

- No space constraints or color figure charges

- Immediate publication on acceptance

- Inclusion in PubMed, CAS, Scopus and Google Scholar

- Research which is freely available for redistribution 